\title{
Temporal variations of the CaXIX spectra in solar flares
}

\author{
R. Falewicz ${ }^{1}$, P. Rudawy ${ }^{1}$, and M. Siarkowski ${ }^{2}$ \\ 1 Astronomical Institute of Wrocław University, 51-622 Wrocław, ul. Kopernika 11, Poland \\ e-mail: [falewicz; rudawy] @astro.uni.wroc.pl \\ 2 Space Research Centre, Polish Academy of Sciences, 51-622 Wrocław, ul. Kopernika 11, Poland \\ e-mail: ms@cbk.pan.wroc.pl
}

Received 29 June 2009 / Accepted 18 September 2009

\begin{abstract}
Aims. The standard model of solar flares comprises a bulk expansion and a rise of abruptly heated plasma (chromospheric evaporation). Emission from plasma ascending along loops rooted in the visible solar disk often should be dominated, at least temporally, by a blue-shifted emission. However, there is only a very limited number of published observations of solar flares having spectra in which the blue-shifted component dominates the stationary one. In this work we compare observed X-ray spectra of three solar flares recorded during their impulsive phases and relevant synthetic spectra calculated using one-dimensional hydro-dynamic numerical model of these flares. The main aim of the work is to explain why many flares do not show blue-shifted spectra.

Methods. We synthetised time series of Bragg Crystal Spectrometer (BCS) spectra of three solar flares at various moments of their evolution from the beginning of the impulsive phases to beyond maxima of the X-ray emission using a 1D numerical model of the solar flares and standard software to calculate BCS synthetic spectra of the flaring plasma. The models of the flares were calculated using observed energy distributions of the non-thermal electron beams injected into the loops, initial values of the main physical parameters of the plasma confined in the loops and geometrical properties of the loops estimated using available observational data. The synthesized BCS spectra of the flares were compared with the relevant observed BCS spectra.

Results. Taking into account the geometrical dependences of the line-of-sight velocities of the plasma moving along the flaring loop inclined toward the solar surface as well as a distribution of the investigated flares over the solar disk, we conclude that the stationary component of the spectrum should be observed for almost all flares during their early phases of evolution. On the contrary, the blueshifted component of the spectrum could not be detected in flares having plasma rising along the flaring loop even with high velocity due to the geometric dependences only. Our simulations based on realistic heating rates of plasma by non-thermal electrons indicate that the upper chromosphere is heated by non-thermal electrons a few seconds before the beginning of noticeable high-velocity bulk motion, and before this time plasma emits the stationary component of the spectrum only. After the start of the upward flow, the blue-shifted component temporally dominates the synthetic spectra of the investigated flares in their early phases.
\end{abstract}

Key words. Sun: chromosphere - Sun: corona - Sun: flares - Sun: magnetic fields - Sun: X-rays, gamma rays

\section{Introduction}

Solar flares are powered mainly by high-energy non-thermal electrons accelerated in the solar corona which stream along magnetic field lines toward the chromosphere, and even the photosphere, and deposit there its energy, mostly in Coulomb collisions. However, a small fraction of the energy carried by non-thermal electrons is converted into hard X-rays (HXR) by bremsstrahlung processes (Brown 1973). Rapid deposition of energy by the electron beams cause the energy not to be radiated away sufficiently fast, therefore a strong pressure imbalanced develops, and heated plasma expands into the corona in a process known as chromospheric evaporation (Antonucci et al. 1984; Fisher 1985; Antonucci et al. 1999).

The heated and evaporated plasma radiates over a wide spectral range from hard $\mathrm{X}$-rays or gamma rays to radio emission, but most of the energy is emitted in soft X-rays (SXR) (Lin \& Hudson 1971; Petrosian 1973). Hard and soft X-ray fluxes emitted by solar flares are generally relate, in a way first described by Neupert (1968), who found that the time derivative of the soft X-ray flux approximately matches the microwave flux during the flare impulsive burst. A similar effect was also observed for hard X-ray emission (Dennis \& Zarro 1993). Since hard X-ray and microwave emissions is produced by non-thermal electrons and soft X-rays are generated by thermal emission from hot plasma, the Neupert effect suggests that non-thermal electrons are the primary source of plasma heating.

Detailed studies (Dennis \& Zarro 1993; Veronig et al. 2002) show deviations from the Neupert effect, indicating that the processes involved are much more complicated. There is clear evidence of an SXR rise before the impulsive HXR emission in some flares (often referred to as preheating), a persistence of the increase and a slower than expected decrease of the SXR flux is frequently observed after the end of the HXR emission. Statistical studies (e.g., Lee et al. 1995; Veronig et al. 2002) indicate that additional heating processes are necessary.

While the mechanisms and processes involved in an abrupt heating of the flaring plasma are still not fully understood, a potentially fruitful way to study solar flare physics is through a comparison of the observational imaging and spectral data with the results of the numerical modeling of the relevant processes.

Spectra of hot and rapidly rising plasma emission are temporally dominated by a blue-shifted component (i.e. blue-shifted emission of the plasma dominates or is comparable to the stationary one). It seems that the strongly blue-shifted emission should be routinely detected during flares, at least during large ones. Most of the papers describing Bragg Crystal Spectrometer (BCS) analysis results indicate that the BCS spectra are 

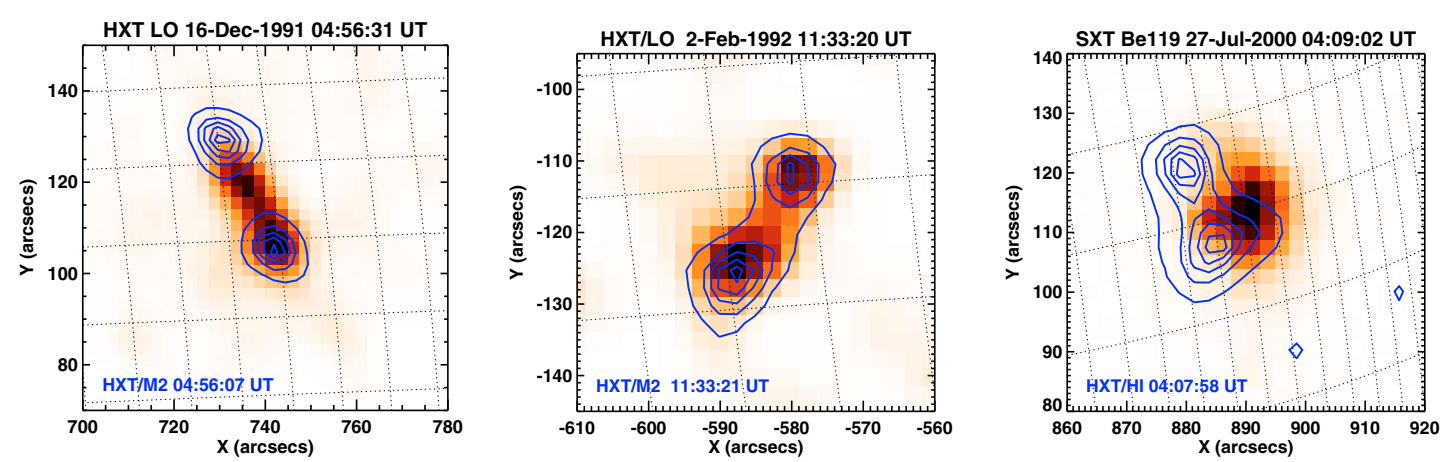

Fig. 1. Images of three analysed flares taken by the Yohkoh satellite with SXT or HXT/LO (gray scale images) and HXT (iso-contours) telescopes.

Table 1. Physical parameters of the analysed flares.

\begin{tabular}{ccccccccc}
\hline \hline $\begin{array}{c}\text { Event } \\
\text { date }\end{array}$ & $\begin{array}{c}\text { Time of } \\
\text { maximum } \\
{[\mathrm{UT}]}\end{array}$ & $\begin{array}{c}\text { GOES } \\
\text { class }\end{array}$ & $\begin{array}{c}\text { GOES } \\
\text { incremental } \\
\text { class }\end{array}$ & $\gamma$ & $a_{0}$ & $E_{\mathrm{c}}$ & $S$ & $L_{0}$ \\
{$\left[\mathrm{ph} / \mathrm{cm}^{2} / \mathrm{s} / \mathrm{keV}\right]$} & {$[\mathrm{keV}]$} & {$\left[10^{17} \mathrm{~cm}^{2}\right]$} & {$\left[10^{8} \mathrm{~cm}\right]$} \\
\hline 16-Dec.-91 & $04: 58$ & M 2.8 & M 2.7 & 3.61 & $7.57 \times 10^{6}$ & 25.8 & 3.85 & 15.40 \\
02-Feb.-92 & $11: 34$ & C 5.5 & C 2.7 & 2.97 & $2.40 \times 10^{5}$ & 18.9 & 2.30 & 09.75 \\
27-Jul.-00 & $04: 10$ & M 2.5 & M 2.4 & 3.20 & $4.79 \times 10^{5}$ & 19.8 & 2.31 & 08.69 \\
\hline
\end{tabular}

$\gamma$ - photon spectral index; $E_{\mathrm{c}}-$ low energy cut-off; $a_{0}-$ scaling factor (flux at $1 \mathrm{keV}$ );

$S$ and $L_{0}-$ cross-section and semi-length of the flaring loop.

dominated by the emission of the stationary component. There is only a very limited number of described observations of solar flares having spectra in which the blue-shifted component dominates the stationary one (e.g., Gan \& Li 2002 and references therein; Culhane et al. 1994). Gan \& Watanabe (1997) performed a statistical study of flares having a single loop structure or a single loop dominating the SXT images. They found that although the blue asymmetry is very common during the impulsive phase, the number of events with large blue-shifts is very small. They also found that for most flares the blue-shift emission appears during early impulsive phases and is temporally correlated with the line broadening. There also exist interesting observations of a blue-shifted emission detected during the gradual phases of flares, although this is out of the scope of this work (Czaykowska et al. 1999).

Most of the published observations of solar events do not indicate the presence of a large blue-shifted component of the spectrum. However, most 1D hydrodynamic (HD) numerical models of chromospheric evaporation in a single loop show the presence of very high up-flow velocities, up to several hundred kilometers per second (Emslie et al. 1992; Mariska \& Zarro 1991; Mariska et al. 1993; Mariska 1994). This discrepancy between observations and models could be explained by insufficient sensitivity of the instruments (Antonucci et al. 1987; Doschek \& Warren 2005), but most of the theoretical works present models of solar flares having low up-flow velocities. The most advanced model of this kind is a multi-thread model of a flare proposed by Hori et al. (1998) and developed by Doschek and Warren, consistent with the observed BCS spectra (Doschek \& Warren 2005; Warren \& Doschek 2005). In this paper, we present another explanation for the lack of the blue-shifted spectra. For three solar flares, we show that one can explain the observed spectra using simple 1D single-loop HD models and taking into account geometrical effects of the viewing angles to the loops.

We describe the observed flares (Sect. 2), the model calculation (Sect. 3), the obtained results (Sect. 4) and provide the discussion and conclusions in Sect. 5.

\section{Observational data}

We selected three solar flares observed by the Yohkoh satellite on the disk, having a clearly recognizable single-loop geometric structure and maximum hard X-ray flux of not less than $10 \mathrm{ctns} / \mathrm{s}$ per subcollimator in the M 2 channel (33-53 keV) of the HXT instrument (Kosugi et al. 1991). Two analysed flares: the C 2.7 GOES class flare observed on 1992 February 2 and the M 2.4 flare on 2000 July 27 were not mentioned previously in the literature; the third one, an M 2.7 class flare on 1991 December 16, was investigated previously by Culhane et al. (1994). All the flares were observed with the HXT telescope during their impulsive phases but only one of them (2000 July 27) was also observed with the Yohkoh SXT grazing-incidence telescope (Tsuneta et al. 1991). HXT images of the flares were reconstructed using the standard Pixon method (Metcalf et al. 1996) with variable accumulation times and an assumed threshold count rate of 200 counts in the M 2 band (33-53 keV). The emission of the flares was routinely recorded with the GOES X-ray photometers (1-8 $\AA$ and $0.5-4 \AA$ bands). The images of the investigated flares are shown in Fig. 1, and their main characteristics are given in Table 1.

Geometric parameters of the loops i.e. their length $\left(L_{0}\right)$, cross-section $(S)$ and inclination were evaluated under the assumption that the observational error of the position of the observed structure is of the order of one pixel (i.e. 2.45 arcsec). In the course of the calculations, both semi-lengths and crosssections of the loops were refined (in a range of the error only) in order to obtain the best conformity between theoretical and observed GOES and BCS light curves (Table 1 presents the final values of $S$ and $L_{0}$ used in the calculations). The errors of inclination of the loops depend on the flare localization on the Sun but for all investigated events is less than $\pm 5 \mathrm{deg}$.

The observed X-ray spectra of the flares were recorded with the BCS instrument on board the Yohkoh satellite (Culhane et al. 1991). The BCS is a full-Sun crystal spectrometer, which measured spectra in the vicinity of strong resonance lines of H-like FeXXVI and He-like FeXXV, CaXIX, and SXV ions using 
curved crystals. The spectra were recorded in one-dimensional position-sensitive proportional counters, with a time resolution of $<1 \mathrm{~s}$. In this paper, we used spectra measured around the CaXIX resonance line. The BCS does not have an absolute wavelength calibration because the location of spectra on the detector depends on the location of the flares on the Sun. To take this pointing offset into account, we determined the rest wavelengths of the spectra using spectra recorded late in the flare when the expected velocities are small.

\subsection{M2.7 flare on 1991 December 16}

The M2.7 GOES incremental class (i.e., background subtraction) solar flare occurred at 04:54 UT on 1991 December 16 in the active region NOAA 6961 on N04W45. The flare appeared as a single loop of moderate semi-length of about $15400 \mathrm{~km}$ in SXR (see Fig. 1). The impulsive HXR emission of the flare above $23 \mathrm{keV}$ was recorded between 04:55:50 UT and 04:56:28 UT. We estimated the cross-section of the loop as equal to $S=3.85 \times 10^{17} \mathrm{~cm}^{2}$ using the reconstructed HXR images.

The BCS observations of this flare were described in detail by Culhane et al. (1994). We used spectra measured around the CaXIX resonance line. The spectra were recorded in a $6 \mathrm{~s}$ cadence from 04:56:06 UT except for the first spectrum recorded at 04:55:42 UT with a $24 \mathrm{~s}$ integration time. A blue-shifted, highly asymmetric component dominated between 04:56:06 and 04:56:48 UT. High asymmetry suggests a wide spectrum of upflow velocities.

A numerical model of the flare was calculated using parameters of the photon spectra $\gamma$ and scaling factors $a_{0}$ calculated as a function of time on the basis of hard X-ray fluxes observed in M 1 and M 2 channels of the HXT instrument.

\subsection{C2.7 flare on 1992 February 02}

The C 2.7 GOES incremental class flare on 1992 February 02 was observed between 11:33 UT and 11:38 UT in an active region NOAA 7042 on S11E41 with Yohkoh/HXT and GOES only (see Fig. 1). The impulsive HXR emission above $23 \mathrm{keV}$ was recorded between 11:33:18 UT and 11:33:28 UT only. HXT/LO images of the flare show X-ray emission of a single loop of moderate semi-length $\sim 9750 \mathrm{~km}$. The feet of the loop were seen in images taken in HXT channels M 2 and $\mathrm{H}$ only. The estimated area of the loop cross-section was $S=2.30 \times 10^{17} \mathrm{~cm}^{2}$.

The CaXIX spectra were recorded from 11:33:01 UT with a 6 s cadence. The blue-shifted component with a velocity higher than $200 \mathrm{~km} \mathrm{~s}^{-1}$ became visible $6 \mathrm{~s}$ later.

The numerical model of the flare was calculated in the same way as for the previous one.

\subsection{M2.4 flare on 2000 July 27}

The M 2.4 GOES incremental class solar flare on 2000 July 27 was observed between 04:08 UT and 04:13 UT in the active region NOAA 9090 on N10W72. The flare was visible in SXT as a single loop of moderate semi-length of about $8690 \mathrm{~km}$ (see Fig. 1). The impulsive hard X-ray emission above $23 \mathrm{keV}$ was recorded between 04:07:54 UT and 04:08:30 UT. We estimated the cross-section of the loop as equal to $S=2.31 \times 10^{17} \mathrm{~cm}^{2}$ using the reconstructed HXR images.

The spectra were recorded with a $6 \mathrm{~s}$ cadence starting from 04:08:11 UT except for the first spectrum recorded at 04:07:47 UT with a $24 \mathrm{~s}$ integration time. From the beginning of the impulsive phase, spectra reveal a clear blue-shifted component. The blue-shifted component was highly asymmetric and dominated between 04:56:06 UT and 04:56:48 UT. This suggests a wide spectrum of up-flow velocities.

The numerical model of the flare was calculated in the same way as for the previous events.

\section{Method of analysis}

The BCS CaXIX synthetic spectra of the investigated events were calculated using results of the 1D numerical HD models of the observed events. The HD models take into account the main factors forming an energy budget and plasma kinetics of the flares: observed energy distributions of the non-thermal electrons and temporal variations of the emitted X-ray fluxes, geometry of the flaring loops estimated using images of the events, estimated main initial physical parameters of the plasma (density, temperature) and processes of gain and loss of energy by the matter.

In this work, we used the modified Naval Research Laboratory Solar Flux Tube Model code kindly made available to the solar community by Mariska and his co-workers (Mariska et al. 1982, 1989). A typical flaring loop can be modeled for many purposes with a simple $1 \mathrm{D}$ hydrodynamic model (as with the NRL Code), although it is a 3D structure surrounded by a complex active region. We included a few modifications to the original NRL code: new radiative loss and heating functions; the VAL-C model (Vernazza et al. 1981) of the initial structure of the lower part of the loop (extended down using Solar Standard Model data; Bahcall \& Pinsonneault 2004), double precision of the calculations and a mesh of new values of the radiative loss function, calculated using the CHIANTI (version 5.2) software (Dere et al. 1997; Landi et al. 2006) for a temperature range $10^{4}-10^{8} \mathrm{~K}$ and density range $10^{8}-10^{14} \mathrm{~cm}^{3}$. All changes were tested by comparison of the obtained results with the results of original models published by Mariska.

An important problem seen during the modeling of the flares using the original NRL code was an insufficient amount of matter located in the feet of the loops. All our attempts to apply energy flux derived from observational data of medium or large solar flares caused massive evaporation of the whole NRL isothermal "chromosphere" and an un-physical "diving" of the feet, even if the chromosphere was unrealistically thick. To solve this problem we applied the VAL C model of the solar plasma, extended using Solar Standard Model data. It was done solely in order to obtain enough storage of the matter. Under such an assumption it does not matter much which particular VAL model is used. All other aspects of the NRL model of the chromosphere are unchanged (radiation is suppressed, optically thick emission is not accounted for, and no account is taken of neutrals).

The main geometrical parameters of the flaring loops: the volume $(V)$, loop cross section $(S)$, half-length $\left(L_{0}\right)$ and a local inclination of the loop's axis to the line of sight were determined using images taken with SXT and HXT. The loop cross sections were estimated as the areas within a flux level equal to $30 \%$ of the maximum flux in the HXT/M 2 channel. Loop half lengths $L_{0}$ were estimated using distances between the centres of gravity of the HXT/M 2 footpoints, assuming a semi-circular shape for the loop an fixed cross-section.

Temperatures $\left(T_{\mathrm{e}}\right)$ and emission measures $(\mathrm{EM})$ of the plasma were estimated using GOES $1-8 \AA$ and $0.5-4 \AA$ fluxes and the filter-ratio method proposed by Thomas et al. (1985) and updated by White et al. (2005). Mean electron densities 

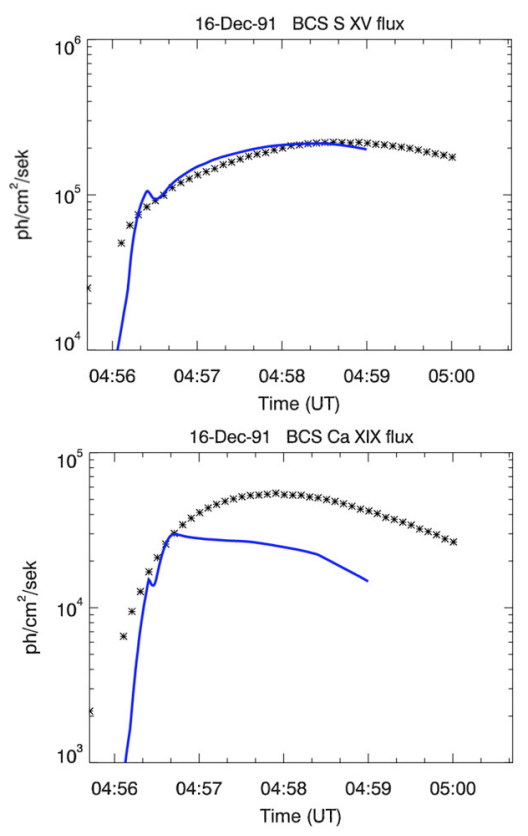

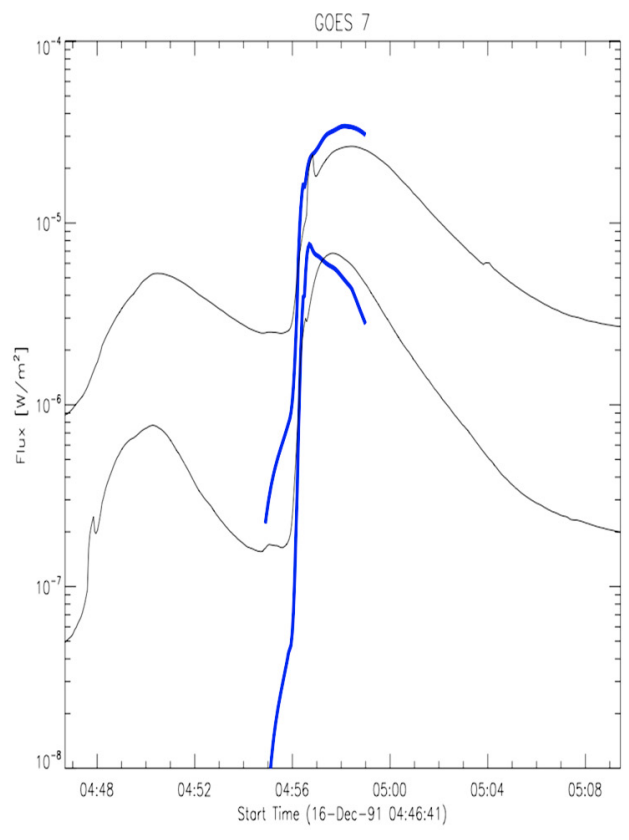

Fig. 2. Left: synthesised BCS CaXIX and BCS SXV fluxes (blue lines), and the observed ones (asterisks) of the M2.7 class solar flare observed on 16 December 1991. Right: synthetic $\mathrm{X}$-ray fluxes in $0.5-4 \AA$ and $1-8 \AA$ bands calculated using a numerical model for the same solar flare observed on 16 December 1991 (blue thick line) and relevant fluxes recorded with the GOES 7 satellite (thin gray lines). $\left(n_{\mathrm{e}}\right)$ were estimated from emission measures (EM) and volumes $\left(V=2 L_{0} S\right)$.

The heating of the plasma by the Coulomb collisions of the non-thermal electrons was modeled using the approximation given by Fisher (1989), necessary parameters of the nonthermal electron distributions were calculated as a function of time from hard X-ray fluxes observed in the HXT M 1 and M 2 channels. We calculated time variations of the spectral indexes $(\gamma)$ and scaling factors $a_{0}$ (photon flux at $1 \mathrm{keV}$ ) for the impulsive phases of the investigated flares assuming power-law hard $\mathrm{X}$-ray photon spectra. Electron spectra of the form $F=A E^{-\delta}$ can be calculated from power-law photon spectra using the thick target approximation. A detailed description of this numerical model is also given by Falewicz et al. (2009).

All models were calculated for periods lasting from the beginning of the impulsive phase beyond the soft X-ray emissions maximum. The time steps in the models were about $0.0005-0.001 \mathrm{~s}$.

While the chromospheric evaporation is powered by nonthermal electrons, a proper estimation of the energy flux carried by electrons is decisive for realistic evaluation of the plasma velocity field. The total energy carried by the non-thermal electrons is very sensitive to the assumed low energy cut-off of the electron spectrum $\left(E_{\mathrm{c}}\right)$, due to the power-law nature of the energy distribution. A change of the $E_{\mathrm{c}}$ value by just a few $\mathrm{keV}$ can add or remove a substantial amount of energy to/from the modelled system, so $E_{\mathrm{c}}$ must be selected with great care. For the investigated flares, we estimated $E_{\mathrm{c}}$ using an iterative method, selecting the $E_{\mathrm{c}}$ value which gives the best agreement of the synthesized and observed X-ray fluxes recorded with GOES X-ray photometers (i.e. GOES classes for 1-8 $\AA$ band) and Yohkoh/BCS fluxes. Examples of the achieved conformity of the observed and synthesized BCS light curves and the observed and synthesized GOES curves are shown in Fig. 2. For each flare the selected value of the $E_{\mathrm{c}}$ was fixed while the electron spectral index $(\delta)$ and corresponding scaling factor $(A)$ varied in time in accordance with the observed variations of the HXR energy flux. The values of the $\delta$ and estimated $E_{\mathrm{c}}$, observed at the maximum of the HXR emission, are given in Table 1.
As a result of HD numerical modeling, we obtain time sequences of 1D models of flaring loops. Each model consists of 1000 cells with individual $T_{\mathrm{e}}$, EM and velocity $v$. For each cell we calculated the corresponding spectrum in the vicinity of the CaXIX resonance line using Chianti 5.2 emissivities for continuum and lines; each cell's spectrum was Doppler-shifted in wavelength $(\lambda)$ by $\Delta \lambda=\lambda \cdot v / c$ according to the local line-ofsight velocity component $(v)$. The total CaXIX spectrum from the loop at given moment of evolution was calculated as a sum of those Doppler-shifted cell's spectra. For better comparison with observations we used the same dispersion i.e., the wavelength bin width as obtained from BCS data.

Velocities projected along the line-of-sight (LOS) for each volume element (cell) of the loop were calculated in the way similar to the method described by Li et al. (1989). The LOS velocities depend on the geometry of the loop, on their orientation (inclination to the solar surface $(\beta)$ and the tilt angle $(\omega)$ ), and their heliographical coordinates $(\lambda, \phi)$. In Fig. 3 we compared spectra obtained for the same velocity distribution along the loop but for two different loop positions on the solar disk. Depending on the loop location and orientation, the blue-shifted component may be dominant or only a small feature in the spectrum.

The inclinations of the loops to the solar surface $(\beta)$ were calculated under the assumption of a semi-circular shape. Using SXT or HXT/LO images, we fitted the observed loops with semicircular loops having the same length and rooted in the same points, but inclined to the solar surface (an example is shown in Fig. 4). Using known longitudes and latitudes of the feet and inclination of the loop to the solar surface, an actual projection of a velocity vector along the loop on the line of sight axis was evaluated for all points of the calculation mesh along the loop.

All investigated flares emitted a slightly enhanced BCS emission long before the beginning of the detected hard X-ray emission. In order to generate a similarly enhanced BCS emission in our numerical models of the flares, we started calculations of the temporal evolution of the models tens of seconds before the beginning of the recorded hard X-ray emission (i.e., before the beginning of plasma heating by non-thermal electrons), applying low energy pre-heatings fitted to mimic the observed enhancement of the BCS emission. The values of the flux maxima are 
$\omega=45, \beta=0, \lambda=0, \varphi=0$

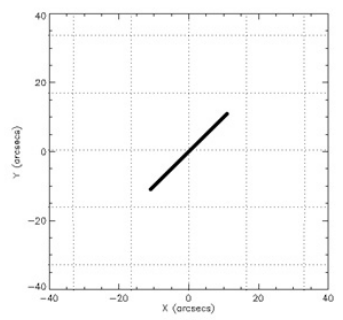

$\omega=45, \beta=0, \lambda=60, \varphi=30$

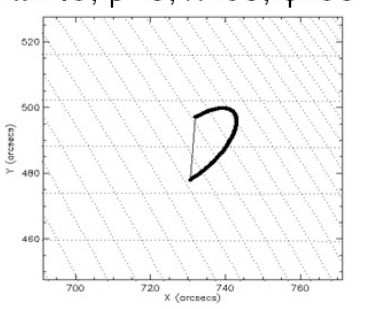

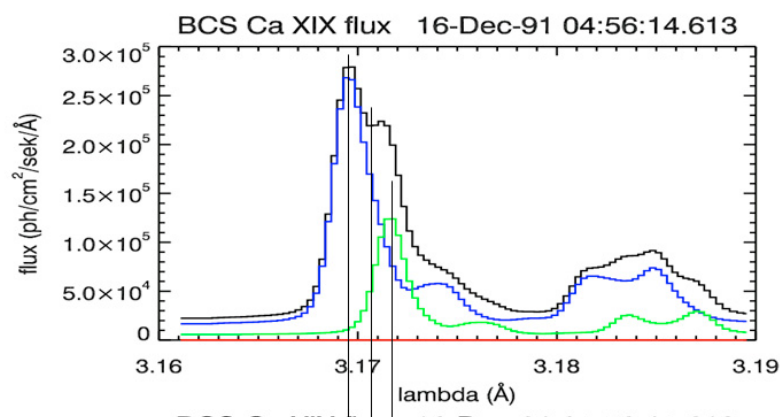

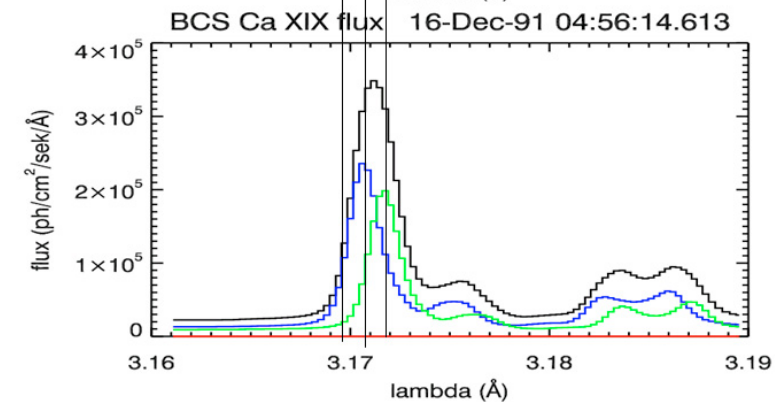

Fig. 3. Influence of the position and orientation of the loop on the spectrum obtained and the observed blue-shift for the same physical and geometrical parameters of the loop. Numerically modeled BCS CaXIX spectra are plotted with solid thin lines (black), the blueshifted components of the modeled spectra are plotted with blue lines, red-shifted components with red lines and the static component with green lines.

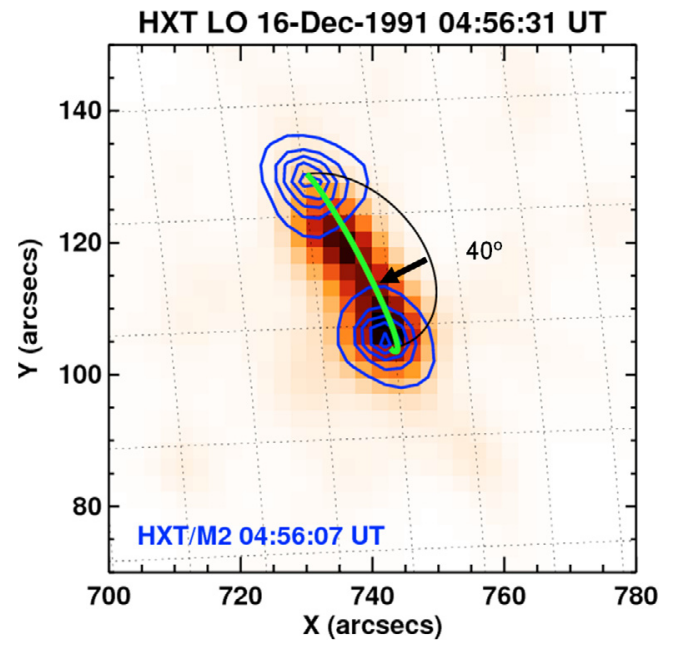

Fig. 4. Image of the flare taken by the Yohkoh satellite with SXT or HXT/LO (gray scale images) and HXT (iso-contours) telescopes. The theoretical semi-circular loop anchored in the footpoints and perpendicular to the solar surface (black) should be declined (tilted) 40 degrees from the vertical to cover the observed loop (green).

reproduced very well but the noticeably large discrepancies during the early rise and especially late decay phases are caused by a lack of additional pre- and post-impulsive heating of the plasma in our model.

The rapid time variations of the energy fluxes carried by nonthermal electrons (of sub-second time scales) cause significant changes of the temperature, density and plasma velocity distributions along the flaring loops and variations of the HXR emission of the solar flares on a sub-second time scale (e.g., Radziszewski et al. 2007). Due to the technical features of the Yohkoh/BCS spectrometer and limited intensities of the X-ray emission of the investigated flares, an effective time resolution of the recorded BCS spectra was of the order of 4-6 s. Thus, each recorded BCS spectrum could be recognized as a sum of numerous spectra emitted consecutively by the flaring loop in the course of the period of the spectrum's accumulation. In order to mimic the process of observed spectra accumulation, the momentary BCS spectra calculated for consecutive time steps of the numerical models of the flares were averaged over the periods equal to the relevant periods of accumulation of the observed BCS spectra. In Fig. 5 we present an example of evolution of the synthetic spectra during accumulation period. These variations are large, both stationary and blue-shifted components can change significantly during the period when BCS collected the spectra. This fact should be kept in mind when interpreting the observed spectra. This is especially important during rising phase of the flares when, because of low statistics, the accumulation times varied from 6 to $9 \mathrm{~s}$.

\section{Results}

We defined blue-shifted, static and red-shifted components of the numerically modeled spectrum as emissions of plasma having radial velocities greater than $50 \mathrm{~km} \mathrm{~s}^{-1}$ (toward the observer), between $-50 \mathrm{~km} \mathrm{~s}^{-1}$ and $50 \mathrm{~km} \mathrm{~s}^{-1}$, and greater than $-50 \mathrm{~km} \mathrm{~s}^{-1}$ (away from the observer), respectively. The observed BCS CaXIX spectra are plotted using solid thick (yellow) lines, numerically modeled spectra are plotted with solid thin lines (black), the blue-shifted component of the modeled spectra are plotted with blue lines, the red-shifted component with red lines and the static component with green lines (see Fig. 6).

\subsection{M2.7 flare on 1991 December 16}

The flare appeared in SXR as a single loop with a semi-length of $15400 \mathrm{~km}$ inclined to the local vertical direction by $40 \mathrm{deg}$ (see Fig. 1, left panel and Fig. 4). BCS observations revealed the strong and highly asymmetric blue-shift component recorded from the beginning of the impulsive phase. The high asymmetry of the spectral lines indicated a wide spectrum of up-flow velocities.

The numerical model of the flare was calculated for the period from 04:54:52 UT to 04:58:52 UT (240 s), which covers the impulsive phase of the flare and spreads beyond the maximum of the recorded GOES satellite emission.

The blue-shifted emission is less than or comparable to the stationary component only in the first observed spectrum. The 


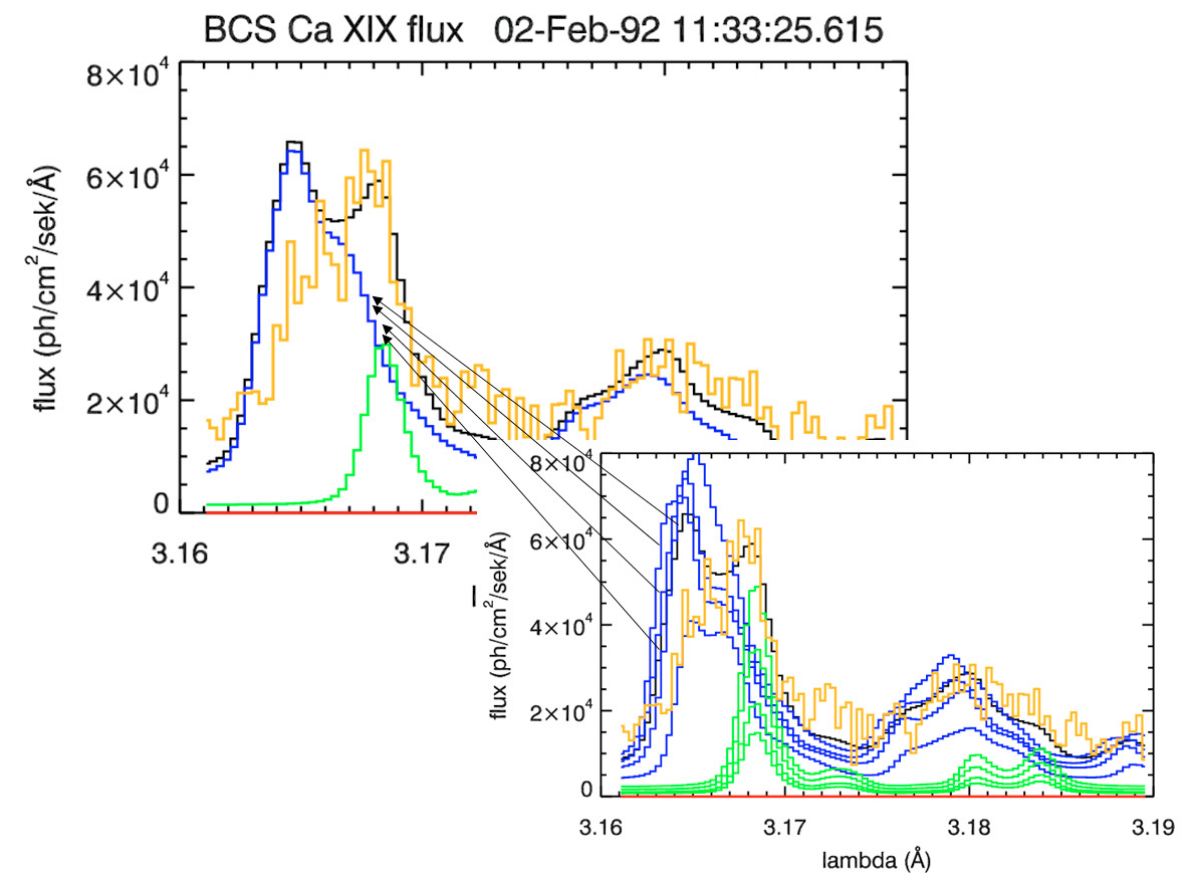

Fig. 5. The observed spectrum as the sum of several spectra from the model. More details can be found in the text. time evolution of the synthetic and observed BCS CaXIX spectra is very similar. The synthetic spectrum consists of the static component only up to 04:56:00 UT. The blue-shifted emission appeared at 04:56:00 UT and increased instantly, but up to 04:56:08 UT it is dominated by the static component. After that, the spectrum was dominated entirely by broadened blue-shifted emission. The observed (yellow) and synthetic (black) spectra recorded between 04:56:12 UT and 04:56:18 UT (6 s accumulation time) as well as blue-, red- and stationary (green line) components of the synthetic spectra are shown in Fig. 6 upper left panel. While the blue-shifted synthetic component is narrower than the much more dispersed observed one, the overall agreement between model and observations seems to be fulfilled. The blue-shifted emission diminished gradually after 04:54:30 UT. Faint red-shifted emission appeared in the synthetic spectra between 04:56:24 UT and 04:56:29 UT. In Fig. 6 (right panel), we compared the observed and synthetic spectra for the period between 04:56:30 UT and 04:56:36 UT (with 6 s accumulation time). Again, the qualitative agreement between theory and observations is good. The blue-shifted component vanished entirely after 04:56:46 UT ( 20-30 s earlier than observed), and the BCS CaXIX spectrum became static again.

\subsection{C2.7 flare on 1992 February 2}

HXT/LO images of the flare show the X-ray emission arriving from a single loop with a semi-length $\sim 9750 \mathrm{~km}$, inclined to the local vertical direction by $35 \mathrm{deg}$ (see Fig. 1, central panel).

The numerical model of the flare was calculated for the period from 11:32:32 UT to 11:35:57 UT (205 s), covering an impulsive phase and spreading beyond the maximum of the emission recorded with GOES. The stationary component was present in both synthetic and observe spectra up to 11:33:01 UT only. There is a good compliance between modeled and observed spectra although the registered signal was weak. After 11:33:13 UT the blue-shifted component with a velocity $v \sim$ $250 \mathrm{~km} \mathrm{~s}^{-1}$ appeared in the spectra. Initially weak, this component increased instantly.
After 11:33:19 UT the blue-shifted component dominated the stationary one. The velocity of blue-shifted component was about $v \sim 220 \mathrm{~km} \mathrm{~s}^{-1}$. There is good agreement between model and observe spectra (see Fig. 6 - left panel, where the color line markings are the same as in earlier events). The stationary component dominates from 11:33:31 UT again and the mean velocity of the blue-shifted component decreased to $v=$ $190 \mathrm{~km} \mathrm{~s}^{-1}$. The time evolution and shapes of synthetic and observed spectra match well. The blue-shifted emission in the modeled spectrum diminished gradually after 11:33:36 UT, vanishing entirely at 11:33:43 UT, when the synthetic BCS CaXIX spectrum became static again. No red-shifted emission was present during the whole modeled period of the flare's evolution.

\subsection{M2.4 flare on 2000 July 27}

The flare was visible in SXT as a single loop perpendicular to the solar surface (see Fig. 1, right panel) with semilength $\sim 8690 \mathrm{~km}$.

The numerical model of the flare was calculated for the period from 04:07:56 UT to 04:10:30 UT, covering an impulsive phase and maximum of the emission recorded with GOES. The observed BCS CaXIX spectra include blue-shifted and stationary components up to 04:07:46 UT. The blue-shifted component with velocity $v=180 \mathrm{~km} \mathrm{~s}^{-1}$ (which is two times weaker than stationary) can be seen in the observed spectra. The model agrees well with the observed spectra although the observed lines are more broadened that the ones in the model. The blueshifted component with velocity $v=290 \mathrm{~km} \mathrm{~s}^{-1}$ dominates in the spectra after 04:08:10 UT. There is a good agreement between modeled and observed spectra. At this time a faint redshifted emission also was present in the synthetic spectra (Fig. 6 - left panel, the color of the lines are the same as in earlier events). After 04:08:16 UT the stationary component begins to dominate again and the velocity of blue component decreased $\left(v=120 \mathrm{~km} \mathrm{~s}^{-1}\right)$, until its complete disappearance (see Fig. $6-$ right panel). A faint red-shifted emission was still present in the synthetic spectra. After 04:08:43 UT the synthetic BCS CaXIX spectrum become static again. 

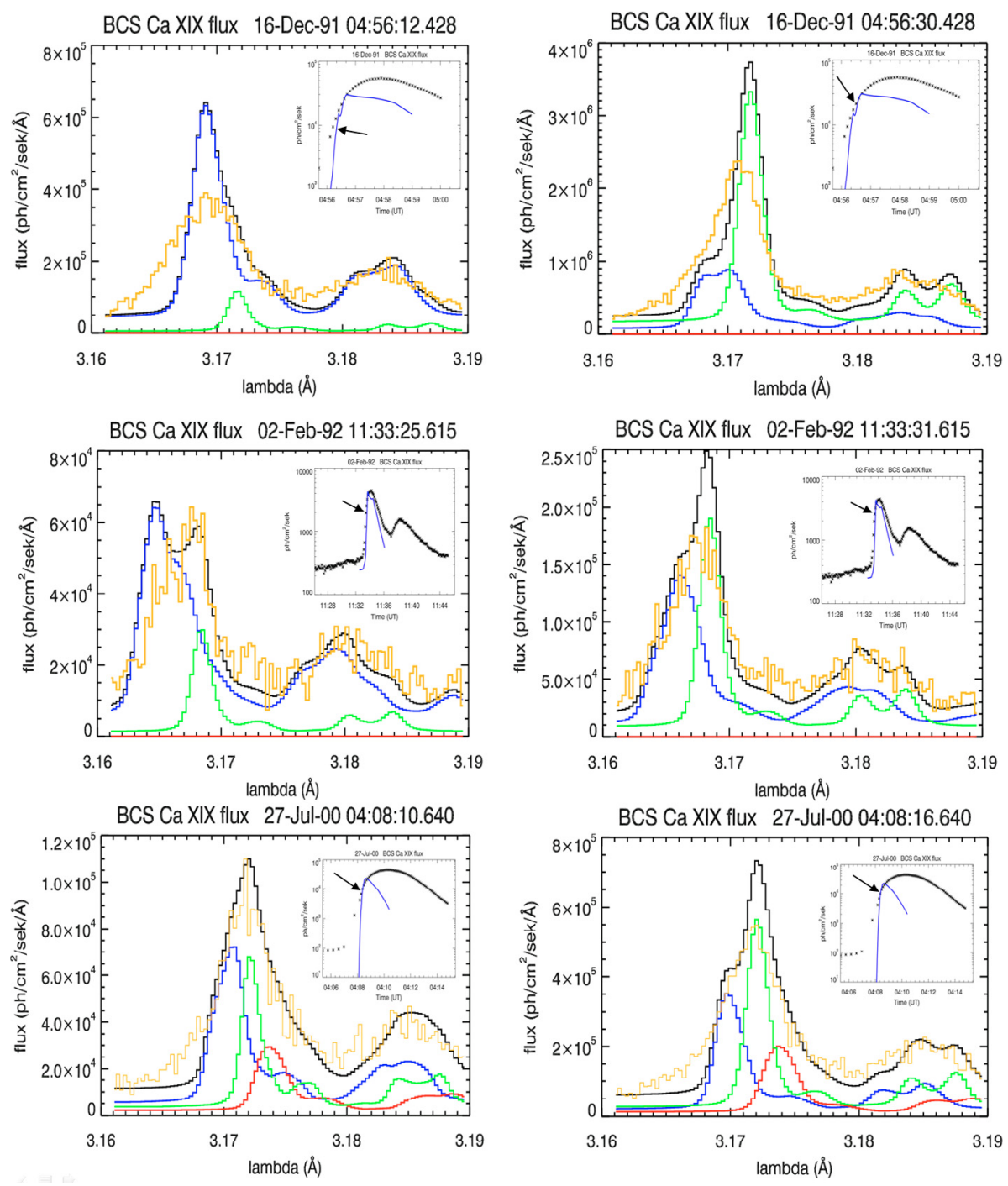

Fig. 6. The observed spectrum for the different moments of time for the three analysed events. Observed BCS CaXIX spectra are plotted using solid thick lines (yellow), numerically modeled spectra are plotted with solid thin lines (black), blue-shifted component of the modeled spectra are plotted with blue lines, red-shifted component with red lines and static component with green lines, respectively.

\section{Discussion and conclusions}

Numerical modeling of the flares allow us to study distributions of the plasma velocities along the flaring loops. Thus, it is possible to estimate the relative contributions of the emissions of the stationary and moving plasma to the spectra (i.e. their stationary and shifted components).

Most papers describing results of the analysis of the BCS data indicate that the BCS spectra are dominated by the stationary component. Additionally, there is only a very limited number of described observations of solar flares having spectra in which the blue-shifted component dominates the stationary one. The statistical paper by Gan \& Watanabe (1997) shows that although moderate blue asymmetry is very common during the impulsive phase, the number of events having spectra with high blue-shift is very small. They also found that for most flares, the blue-shift emission appears during early impulsive phases and is temporally correlated with the line broadening.

Most 1D numerical models of chromospheric evaporation in single loops show a presence of very fast up-flows, up to several hundred kilometers per second. This discrepancy between observations and models could be explained by insufficient sensitivity of the instruments but most of the theoretical works present models of the solar flare having low up-flow velocities. The most advanced model of this kind is a multi-thread model of the flare proposed by Hori et al. (1998) and developed by
Doschek \& Warren (2005), consistent with the observed BCS spectra. In this paper we present another explanation of the lack of the observed blue-shifted spectra.

We investigated the C 2.7 GOES class flare observed on 1992 February 2, the M 2.4 flare observed on 2000 July 27, and the M 2.7 class flare observed on 1991 December 16. Two of the analyzed flares (C 2.7 and M 2.4) were not described previously in the literature; the third one, M 2.7 was investigated by Culhane et al. (1994). The synthetic BSC spectra of the flares were calculated using real observational parameters of the observed flares (geometry, physical parameters of the plasma, energy spectrum of the non-thermal electrons).

For all investigated events, we obtained good quantitative agreement of the synthetic and observed BCS CaXIX spectra. For the whole impulsive phase of each flare, we obtained overall good agreement of fluxes, shapes and time variations of the observed and synthetic spectra.

Generally, widths of the observed spectral lines were greater than widths of the relevant synthetic spectral lines. The observed blue-shifted component was wider than the synthetic one and highly asymmetric. This suggests a wider dispersion of up-flow velocities in observed events than in our theoretical models. This non-conformability could be caused by disregarded turbulent motions of the plasma in our numerical model and by some differences between calculated and real distributions of the 
physical parameters of the plasma along the loops (densities, temperatures, and macroscopic velocities). Nevertheless, we obtained good agreement between overall shape, and stationary, red- and blue-shifted components of the relevant calculated and observed spectra.

Despite very high up-flow velocities of the plasma obtained in numerical simulations of the various flares, similarly high radial velocities could be observed mostly for the events located in the central part of the solar disc only, due to a geometrical projection of the velocity vector onto the direction of sight. Assuming a semi-circular loop located perpendicularly to the local solar surface and rooted at the solar longitude of $60 \mathrm{deg}$, the line-of-sight (LOS) component of the velocity is two times smaller than the real velocity of the plasma along the loop. Similarly about $50 \%$ of the emission emitted as blue-shifted from the loop rooted at the disc centre is observed as a stationary component for the loop rooted at the solar longitude of $60 \mathrm{deg}$. So, taking into account the geometrical dependences of the LOS velocities of the plasma moving along the loops inclined toward the solar surface as well as the distribution of the flare sites over the solar disc, we can conclude that stationary component can be observed for all flares during their early phases of evolution (i.e., in almost all flares some plasma exists which does not move along the LOS). On the other side, the blue-shifted component of the spectrum could not be detected, even for plasma rising along the flaring loop with very high velocity. Additionally, our simulations based on realistic heating rates by non-thermal electrons indicate that the upper chromosphere plasma is heated by non-thermal electrons a few seconds before the beginning of noticeable high-velocity bulk motion, and until this time it emits a stationary (not red- or blue-shifted) spectrum only.

Very similar investigations of the impact of the various viewing angles on the resulting spectral signature of the flare were made by Li et al. (1989). They used theoretical models of flaring loops with arbitrarily selected plasma parameters and temporal variations of the local heating. For most cases they obtained a strong blue-shifted component of the spectrum. Our models are based on observational data; duration of the heating and delivered energy flux were evaluated from Yohkoh/HXT data. In our models, the absolute velocities of the plasma up-flow were of the order of $450-500 \mathrm{~km} \mathrm{~s}^{-1}$, but taking into account the geometrical effect of the angle of view on the loops, they were reduced to a moderate $200-250 \mathrm{~km} \mathrm{~s}^{-1}$.

Taking into account the geometrical dependences of the line-of-sight velocities of the plasma moving along the flaring loop inclined toward the solar surface as well as the distribution of the investigated flares over the solar disk, we conclude that the stationary component of the spectrum should be observed for almost all flares during their early phases of evolution. The blueshifted component of the spectrum could not be detected in flares having plasma rising along the flaring loop even with high velocity due to the geometrical dependences only. Our simulations based on realistic heating rates of plasma by non-thermal electrons indicate that the upper chromosphere is heated by nonthermal electrons a few seconds before the beginning of noticeable high-velocity bulk motion, and before this time, plasma emits a stationary component of the spectrum only. After the start of the up-flow motion, the blue-shifted component temporally dominates the synthetic spectra of the investigated flares in their early phases.

Acknowledgements. The authors would like to thank the Yohkoh team for excellent solar data and software. They are also grateful to the anonymous referee for useful comments and suggestions. This work was supported by the Polish Ministry of Science and Higher Education, grant number N203 022 31/2991.

\section{References}

Antonucci, E., Gabriel, A. H., \& Dennis, B. R. 1984, ApJ, 287, 917

Antonucci, E., Dodero, M. A., Peres, G., Serio, S., \& Rosner, R. 1987, ApJ, 322, 522

Antonucci, E., Alexander, D., Culhane, J. L., et al. 1999, in The Many Faces of the Sun: a Summary of the Results from NASAs Solar Maximum Mission, ed. K. T. Strong, J. L. R. Saba, B. M. Haisch, \& J. T. Schmelz (Springer-Verlag), chapt. 10.

Bahcall, J. N., \& Pinsonneault, H. M. 2004, Phys. Rev. Lett., 92, 12

Brown, J. C. 1973, Sol. Phys., 31, 143

Culhane, J. L., Hiei, E., Doschek, G. A., Cruise, A. M., et al. 1991, Sol. Phys. 136,89

Culhane, J. L., Phillips, A. T., Inda-Koide, et al. 1994, Sol. Phys., 153, 307

Czaykowska, A., de Pontieu, B., Alexander, D., \& Rank, G. 1999, ApJ, 521, L75 Dennis, B. R., \& Zarro, D. M. 1993, Solar Phys., 146, 177

Dere, K. P., Landi, E., Mason, H. E., Monsignori-Fossi, B. C., \& Young, P. R. 1997, A\&AS, 125, 149

Doschek, G. A., \& Warren, H. P. 2005, ApJ, 629, 1150

Emslie, A. G., Li, P., \& Mariska, J. T. 1992, ApJ, 399, 714

Falewicz, R., Rudawy, P., \& Siarkowski, M. 2009, A\&A, 500, 901

Fisher, G. H. 1989, ApJ, 346, 1019

Fisher, G. H., Canfield, R. C., \& McClymont, A. N. 1985, ApJ, 289, 425

Gan, W. Q., \& Li, Y. P. 2002, Solar Phys., 205, 117

Gan, W. Q., \& Watanabe, T. 1997, Adv. Space Res., 20/12, 2319

Hori, K., Yokoyama, T., Kosugi, T., \& Shibata, K. 1998, ApJ, 500, 492

Kosugi, T., Masuda, S., Makishima, K., et al. 1991, Solar Phys., 136, 17

Landi, E., Del Zanna, G.,Young, P. R., et al. 2006, ApJS, 162, 261

Lee, T. T., Petrosian, V., \& McTiernan, J. M. 1995, ApJ, 448, 915

Li, P., Emslie, A. G., \& Mariska, J. T. 1989, ApJ, 341, 1075

Lin, R. P., \& Hudson, H. S. 1971, Sol. Phys., 17, 412

Mariska, J. T. 1994, ApJ, 434, 756

Mariska, J. T., \& Zarro, D. M. 1991, ApJ, 381, 572

Mariska, J. T., Boris, J. P., Oran, E. S., Young, T. R., Jr., \& Doschek, G. A. 1982, ApJ, 255, 738

Mariska, J. T., Emslie, A. G., \& Li, P. 1989, ApJ, 341, 1067

Mariska, J. T., Doschek, G. A., \& Bentley, R. D. 1993, ApJ, 419, 418

Metcalf, T. R., Hudson, H. S., Kosugi, T., Puetter, R. C., \& Pina, R. K. 1996, ApJ, 466, 585

Neupert, W. M. 1986, ApJ, 153, L59

Petrosian, V. 1973, ApJ, 186, 291

Radziszewski, K., Rudawy, P., \& Phillips, K. J. H. 2007, 461, 303

Tsuneta, S., Acton, L., Bruner, M., et al. 1991, Solar Phys., 136, 37

Vernazza, J., Avrett, E., \& Loeser, R. 1981, ApJS, 45, 635

Veronig, A. M., Vrsnak, B., Dennis, B. R., et al. 2002, A\&A, 392, 699

Warren, H. P., \& Doschek, G. A. 2005, ApJ, 618, L157 